\title{
Lung Structure Classification Using 3D Geometric Measurements and SVM
}

\author{
João Rodrigo Ferreira da Silva Sousa, Aristófanes Corrêa Silva, \\ and Anselmo Cardoso de Paiva \\ Federal University of Maranhão - UFMA \\ Av. dos Portugueses, SN, Campus do Bacanga, Bacanga \\ 65085-580, São Luís, MA, Brazil \\ j.rodrigo.sousa@gmail.com, ari@dee.ufma.br, \\ paiva@deinf.ufma.br
}

\begin{abstract}
In this paper, a set of three features for aiding classification of lung nodule bearing candidates based upon morphological characteristics is proposed. Metrics were validated using Support Vector Machine (SVM) technique as classifier. Preliminary results indicate the efficiency of the adopted measurements, taking into account the sensitivity and specificity high rates obtained from the studied samplings.
\end{abstract}

Keyword: Lung Nodule, Classification, Geometric Measures, SVM.

\section{Introduction}

In the last years lung cancer is gaining attention from the scientific community due to its high occurrence among people and also due to the difficulty to treat it. Nevertheless, it is well known that the principal cause of the illness is associated with the smoking habits, especially among males population, in such a way that the chances for a positive diagnostic increase from 20 to 30 times amongst smokers [1].

Research has been performed in the computer field aiming at producing tools to aid the physician in the analysis of computer tomography imaging (CT). Those tools can be divided into two main groups: lung nodule detection and diagnosis aiding ones.

Lung nodule detection is characterized by the identification, from a set of CT images, of a region presenting features that identify it as lung nodule. To do this, in general, a two fold process is conducted: first, the various structures existing in the parenchyma are segmented; next, the structures are classified as to identify which among them represent the nodules and which are associated to the rest of structures that lay, usually, in the lung parenchyma (non-nodules).

In the area of lung structures classification through CT, significant progress has been observed. Several discriminatory measures have been proposed as to differentiate nodules from non-nodules. There are morphological approaches that use geometrical structure characteristics, both bi-dimensional and threedimensional, to describe them. [2] introduced a new characteristic able to 
estimate the nodule tissue radial distribution in a non binary way, getting promising target hitting [3]; on the other hand, he developed a method for rebuilding lung trees supported on highlighting filters, demonstrating its applicability as an aid tool for nodule detection.

There are, also, works that adopt texture characteristics to describe lung tissue and thus, identify it. In 4, for example, there were used 22 characteristics texture based to classify the regions of a CT image from 6 possible groupings, among which the nodule, bronchial-vascular and normal.

In most of the works, once the structures' representative characteristics have been calculated, classifiers for identifying them within the group of nodule or non-nodules are used. One of the classifiers that became very popular because of its high generalization capacity is the Support Vector Machine (SVM), already used in several researches [5, 2, 6]. Neural networks and grouping algorithms are other very popular type of classifiers, also presenting satisfactory results.

In spite of these developments, however, there is not yet a methodology neither in the segmentation area nor in the classification one, which could be considered as definitive for lung nodules identification. Most of them still need improvements concerning target hitting rates or generalization capacity, making it still an open problem.

In fact, lung structures classification is still facing several difficulties. One of them, for example, says respect to the tissue density, which is very alike among the blood vessels and some kind of nodules, leading the classifier to commit same mistakes.

Another difficulty concerns the malignant nodules form. This kind of nodule usually presents spikes and branching due to its disordered nature. The classification problem derived from this is that these branches are similar to the vessels irrigating the nodule which, in its turn, are also numerous in the case of a malignant lesion.

Thus, a great deal of the problem faced by researchers is linked with the difficulty of proposing measures for describing the lung parenchyma inner structures, in such a way as to allow a maximization of the used classifier target hitting rates. This dilemma can still be aggravated by the fact that, under certain aspects such as tissue density or form, a nodule could be considered similar to a lung's normal structure, confusing the classifier.

Besides the techno-scientific challenges above, this problem presents social motivation due to its close association with human health. Hence, the present work aims at contributing with the automation efforts for detecting lung nodules while proposing some novel discriminatory characteristics for the parenchymal structures using them, associated to a SVM classifier, to compare its efficiency with other traditional approaches.

This work is organized as follows: In Section 2, the methodology and the features proposed for the geometrical description of the structures under analysis is explicated. Next, in Section 3, presents the criteria and parameters used in the analysis of the proposed characteristics, as well as the results obtained with them and their comparison with other studies results. Finally, Section 4 closes 
the study by analyzing the viability of the described features supported by the obtained results.

\section{Methods}

In this Section, the proposed method for lung nodules detection is presented and demonstrated the data base formation process used in trials and method validation. Next, three new classification features based upon objects' morphology is also presented. Finally, the SVM technology, basement of the classifier, is introduced.

\subsection{Pulmonary Structures Segmentation}

The images were acquired with a Helical GE Pro Speed tomography under the following conditions: tube voltage $120 \mathrm{kV}$, tube current $100 \mathrm{~mA}$, image size $512 \times 512$ pixels, voxel size $0.67 \times 0.67 \times 1.0 \mathrm{~mm}$ and reconstruction interval of 1 $\mathrm{mm}$. The images were quantized in $12 \mathrm{bits}$ and stored in the DICOM format [7].

It is important to stand out that the CT exam was performed with no contrast injection, which may be clinically used in order to increase the diagnosis readiness but also carries some morbidity and occasional mortality by allergic complications.

It is also necessary to highlight that the nodules were previously diagnosed by physicians and that the final diagnosis of benignity or malignancy was posteriorly confirmed by histopathological exam of the surgical specimen or by radiologic 3-year stability, which explains the reduced size of our sample.

Nodules were semi-automatically segmented by a specialist physician who used the Lung Nodule Analysis System (Bebúi). This system, proposed in [8, allows the three-dimensional segmentation of the lung nodule by means of a region growing algorithm starting from given seed indicated by the specialist physician. A preliminary segmentation is, next, submitted to the users' approval through the observation of two dimension slices. At this point, if necessary, the physician may manually tune the segmentation as to increase the results accuracy.

Other parenchymal structures, such as blood vessels and bronchia, were automatically segmented through a serial processing of the TC data, under a segment modularizing scheme. This segmentation scheme uses parameters dynamically defined through properties obtained from the images of each examination. This approach avoids the dependency on parameters statically defined which could embarrass the generality of the method.

The entirely automatic processing starts by removing from the bulk the dense tissues involving the lung, mainly composed by muscles and bones, through a selective threshold algorithm by location. Next, by using the morphology technique known as rolling ball, the lung walls are restored as not to neglect peripheral nodules. The next step is to remove from lungs the soft tissues, principal constitutive of the parenchyma, preserving only the structures 
contained in it, what is again performed aided by a dynamical threshold. Finally, by using region growing algorithm, each structure is identified and isolated. Samples of the segmented structures through the described methods can be seeing in Figure 1]. Figures 17 and 10 represent normal lung structures automatically segmented, while the ones of Figures 1rc and 1 id isolated lung nodules segmented by the Bebúi system.

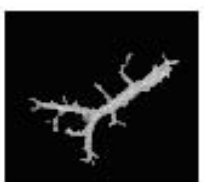

a

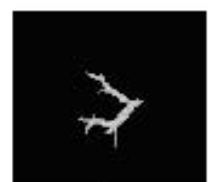

b

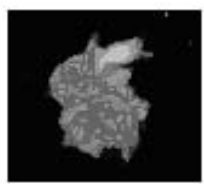

C

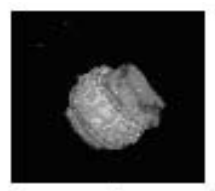

d

Fig. 1. Structures sampling present in the parenchyma. c and d are isolated lung nodules.

Considering both methods, it was possible to build a database with samples corresponding to nodules segmented by the Bebúi system and the rest of the lung parenchymal structures.

In total, 38 samples were used, corresponding to benign and malignant nodules which volume varied between $0.06 \mathrm{~cm}^{3}$ and $88 \mathrm{~cm}^{3}$. There were also used 228 structures belonging to the class non-nodule, among which blood vessels and bronchia.

\subsection{Proposed Features}

As to allow classification and attain satisfactory target hitting rates, it is important to use the under analysis structures' characteristics. Thus, this Section presents the proposal of three new measurements based on the object's geometry (morphology) to be classified.

Spherical Disproportion. Morphologically it is well known that blood vessels are very different than lung nodules, when observed three dimensionally. While the former presents elongated shape, being possible branches formation, the latter presents a compact and round feature.

Conducting the analysis under this aspect, the measurement of the round disproportion can tell us until which extent certain structure presents unsmooth surface with respect to another totally round surface. This is got by means of a comparison between the estimation of the area the volume should have, if it were totally spherical, and its actual area.

It must be noted the difference between border voxels and surface area. The set of border voxels of an object forms a volume, i.e, it is a three dimensional magnitude, while the outside area is a surface, hence a bi-dimensional one.

In such a way, the spherical disproportion is calculated by Equation 1;.

$$
D=\frac{A}{4 \pi R^{2}}
$$


where $A$ is the object's surface area, $R$ is the estimated sphere's radius with the same volume as the object. The estimated radius $(R)$ is obtained by

$$
R=\sqrt[3]{\frac{3 V}{4 \pi}}
$$

where $V$ is the object's volume.

The Spherical Disproportion assumes smaller values for round objects and higher values for irregular or elongated objects. The measurement also presents invariant properties with respect to the rotation, translations and to the scale.

Spherical Density. As already said, the various structures, nodular or not, found at the lung parenchyma, have more or less compact formats according to their own nature. In such a way, more compact structures are very similar to spheres, while less compact structures look differently.

A very ordinary measurement for measuring how compact a structure is consists in comparing its volume with that one of the minimum box [9]. There are, however, variations of this technique through which it is possible to use the convex hull [10] or, yet, other geometrical figures such as ellipses [9]; but in general, the method encloses the calculation of an object's circumscribed figure and compare its volume with the one occupied by the object under study.

These techniques, nevertheless, face the problem of calculating the best object circumscribed figure alignment or inconsistencies related with the lack of alignment, such as different values for identical objects, but with different rotation, that is, they are not rotationally invariant.

The spherical density, on the other hand, uses a sphere, a figure rotationally invariant, but without having to calculate optimal measurements to circumscribe it to the object. What is in fact done is to use a sphere which volume is the same as the one of the object under study, with origin in the object's center of mass. Thus, the measurement of the spherical density will consist in this sphere's percentage that, in fact, also corresponds to the objects'.

Figure 2 visually represents this feature in 2D format, even though the feature is in $3 \mathrm{D}$, as to easy the visualization and the understanding through a media that is also bi-dimensional. Note that, in Figure 2 a, the object occupies most of the circle, resulting in a high spherical density, In Figures $2 \mathrm{~b}$ and 2 c occurs the opposite due to greater dispersion of the object's volume, in such a way that the resulting spherical density assumes smaller values.

Spherical Density is calculated by:

$$
\frac{100 \cdot n}{V}
$$

In this paper, the following notation was used: every time $x, y$ and $z$, together or separately, would appear underlined, it would indicate a point coordinates, whereas whenever they appear in between parenthesis, they will be indexing an antecedent variable with respect to the respective axis in the objects voxels grid. Thus, in Equation $3 . p(x, y, z)$ is the voxel value with coordinates $x, y$ and $z, n$ 


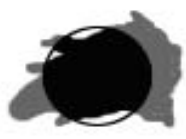

a

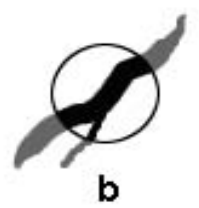

b

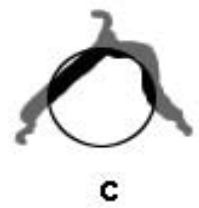

C

Fig. 2. Spherical Density bi-dimensional Illustration

is the amount of voxels $p$ such that $p(x, y, z) \neq 0$ and $\left(x-M_{x}\right)^{2}+\left(y-M_{y}\right)^{2}+$ $\left(z-M_{z}\right)^{2} \leq R^{2}, V$ is the object's volume, $R$ is the estimated radius obtained through Equation 2 and $M_{x}, M_{y}, M_{z}$ is the object's center of mass coordinate.

The Spherical Density presents the property of getting close to zero in very elongated objects or with external mass center, while it assumes values close to 100 for more round volumes, even though the interval $[0,1]$ could be used without affecting the results just by removing the constant in the Equation 3 . Besides this, it is an invariant measurement with respect to changes in the object concerning rotation, translation and scale; being, thus, a very fair stable measurement to be used as a reliable morphological describer.

Weighted Radial Distance. Feature that calculate the flatness or lengthening of an object based on circumscribed images or on global characteristics are flawed because they fail in measuring these objects' real peculiarities.

In Figure 3 one can notice that, even when the three objects minimum boxes factually present the same dimensions, the own objects themselves are very different. Measurements taken upon these boxes' dimensions run the risk of falling into significant lack of accuracy.

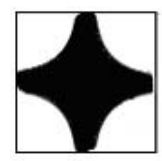

a

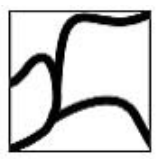

b

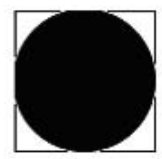

c

Fig. 3. Objects represented in their minimum boxes

Global measurements such as area and volume not always are ideal descriptors for certain objects' characteristics as it is known, again, that those ones holding the same measurements may present very different shapes.

The Weighted Radial Distance ponders the degree of flatness of an object, starting from voxels' local measurements, increasing the overall precision of this descriptor.

The idea behind this measurement lays in the distance relationship between the volume's voxels and its own medial axis. In very elongated volumes, the voxels, in average, tend to be at a short distance from the medial axis, while in more concentrated volumes the voxels assume longer radial distances. 
Nevertheless, the mean radial distance with respect to the medial axis, even when it already supplies an idea of the objects' degree of flatness, is not a consistent measurement, once volume objects will tent to present higher coefficients.

Thus, the Radial Distance is taken in a pondered fashion, so assuring that the volume's dimension will not interfere in the measurement magnitude, but just in its format.

The Weighted Radial Distance can be obtained by means of

$$
P=R^{-1} \sum_{x, y, z} c(x, y, z)
$$

where $R$ is the estimated radius obtained through Equation $2 . c(x, y, z)$ is the weighting coefficient applied to each voxel by means of

$$
c(x, y, z)=\frac{3}{4 \pi}\left[[r(x, y, z)+0,5]^{3}-[r(x, y, z)-0,5]^{3}\right]^{-1}
$$

and $r(x, y, z)$ is the radial distance of a voxel with coordinates $x, y, z$.

The radial distance $r(x, y, z)$, as explained before, is the voxel's distance to medial axis at that point. It is calculated by the inverse distance transform.

Initially, the distance's transform is done in a smooth manner on the volume, assigning to each voxel an index which represents its degree of remoteness from the borders. The most inner voxels, which are part of the medial axis, will present, then, higher values.

The inverse radial distance is but nothing else than the reversal of the ascendant direction of those values, given the value zero to the central voxels and to the remaining ones, as they go away, successively higher values until the borders are reached.

It is important to notice that the same result can not be obtained just from the conventional radial distance calculation based in the origin. This happens because the former is based upon the distance with respect to a single central point, while the radial distance calculated through the described process obtains the voxels distance with respect to the object medial axis, which is formed by a series of points.

The Weighted Radial Distance presents the property of assuming values close to unity in not very elongated volumes, and higher values for more elongated volumes. It is also an invariant measurement for rotations, translations and scales.

\subsection{Support Vector Machine}

The Support Vector Machine (SVM) introduced by V. Vapnik in 1995 is a method to estimate the function classifying the data into two classes [11]. The basic idea of SVM is to construct a hyperplane as the decision surface in such a way that the margin of separation between positive and negative examples is maximized. The SVM term come from the fact that the points in the training set 
which are closest to the decision surface are called support vectors. SVM achieves this by the structural risk minimization principle that is based on the fact that the error rate of a learning machine on the test data is bounded by the sum of the training-error rate and a term that depends on the Vapnik-Chervonenkis (VC) dimension.

In the proposed work, the characteristics obtained from each candidate trough of the Equations 2, 3 and 4 had to be supplied to the classifier in such a way as allowing it to evaluate the classes separation hyper-plane and, next, classify other objects.

Nevertheless, each characteristic varies in different value bands, which would lead to a tendency, by the part of the classifier, for super-estimating the relevance of some of them, due to a greater interval variation. As to avoid this fact to become a problem, it is necessary to proceed with a quantization as to translate all the intervals of each characteristic to a common variation band.

The interval selected in this work was $[-1,1]$, once it is conventionally accepted and used with SVM.

After the quantization of all the intervals, the characteristics obtained in such a way give raise to the $x$ points of the input space.

\subsection{Validation of the Classification Methods}

In order to evaluate the classifier in respect to its differentiation ability, we have analyzed its sensitivity, specificity and accuracy. Sensitivity is defined by $T P /(T P+F N)$, specificity is defined by $T N /(T N+F P)$, and accuracy is defined by $(T P+T N) /(T P+T N+F P+F N)$, where $T P$ is true-positive, $T N$ is true-negative, $F N$ is false-negative, and $F P$ is false-positive. Herein, truepositive means Mass samples were correctly classified as Mass. The meaning of the others are analogous.

\section{Results}

The adopted SVM classifier was the libsvm [12] library configured to use, during classification, the Gaussian RBF kernel due to its well known generalization power.

The target hitting statistical evaluation procedure conducted the samples cross validation, taking for each iteration a pair of two elements arbitrarily grouped for testing purposes, and the remaining ones for training, i.e., the diverse samples compounding the data base were occasionally, and at different iterations, used for both, training and validation, according to the group they belonged at that very moment. This sort of validation allowed the use of all samples as to obtain a more consistent training, as well as a more precise validation.

This procedure for targer hitting evaluation was separately conducted for all three measurements, all together with the three proposed measurements. According within this criterion, it was verified that, among the proposed characteristics, the one who presented the best results was the Spherical Density. 
This feature, alone, was able to attain a rather good target hitting degree, as can be checked in Table 1 .

Table 1. The proposed features comparison

\begin{tabular}{c|c|c|c} 
Features & $\begin{array}{c}\text { Sensitivity } \\
\%\end{array}$ & $\begin{array}{c}\text { Specificity } \\
\%\end{array}$ & $\begin{array}{c}\text { Accuracy } \\
\%\end{array}$ \\
\hline Spherical Disproportion & 78.94 & 100 & 96.99 \\
\hline Spherical Density & 100 & 99.56 & 99.62 \\
\hline Weighted Radial Distribution & 71.05 & 97.36 & 93.60 \\
\hline The three proposed features & 100 & 100 & 100 \\
\hline Radial Volume distribution & 91.40 & 99.5 & -
\end{tabular}

Nevertheless, the most important was that the remaining proposed features, together with the Spherical Disproportion, even though they did not do in isolation such a good classification, characterize the nodule candidate in such a precise way that, the total target hitting rate reached $100 \%$.

Based on the presented results, we have observed that this new methodology provides significant support for a more detailed clinical investigation. Nevertheless, there is the need to perform tests with a larger database and more complex cases in order to obtain a more precise behavior pattern.

For comparison, Table 1 also presents the results obtained from other approaches. The Radial Volume Distribution was proposed by 2, who demonstrated this as having a very high descriptive power.

\section{Conclusion}

This paper presented a set of three geometrical measurements to be used in the discrimination between nodules non-nodules. The results of the classification based upon these measurements were compared with the results obtained from other works, verifying that the proposed measurements present a rather good discrimination power.

It was also confirmed that, once each measurement was destined to describing a morphological aspect different of that one of the candidates, they, in isolation, can not fully characterize them. However, the set of features proved to be fairly efficient in the candidates' description, reaching $100 \%$ correct classification in the test samples.

The size of the samples used for tests, however, was too small for getting any definitive conclusion, but the statistics obtained from the tests, specially the ones concerning the target hitting rate, showed that the proposed measurements appear themselves as viable alternatives for geometrical objects description for a variety of applications, particularly in the medical area for classifying lung nodules. 


\section{Acknowledgements}

The authors acknowledge CAPES (process number 0044/05-9) and CNPq (process number 472104/06-3) for financial support.

\section{References}

1. Partnership, N.L.C.: Frequently asked questions (2007), Available at http://www.nationallungcancerpartnership.org

2. Bi, J., Periaswamy, S., Okada, K., Kubota, T., Fung, G., Salganicoff, M., Rao, R.B.: Computer aided detection via asymmetric cascade of sparse hyperplane classifiers. In: KDD 2006, pp. 837-844. ACM Press, New York (2006)

3. Agam, G., Armato III, S.G., Wu, C.: Vessel tree reconstruction in thoracic ct scans with application to nodule detection. IEEE Trans. Med. Imaging 24, 486-499 (2005)

4. Uppaluri, R., Hoffman, E., Sonka, M., Hartley, P., Hunninghake, G., Mclennan, G.: Computer recognition of regional lung disease patterns. American Journal of Respiratory and Critical Care Medicine 160, 648-654 (1999)

5. Korfiatis, P., Kalogeropoulou, C., Costaridou, I.: Computer aided detection of lung nodules in multislice computed tomography (2006)

6. Mousa, W.A.H., Khan, M.A.U.: Lung nodule classification utilizing support vector machines. In: ICIP (3), pp. 153-156 (2002)

7. Clunie, D.A.: DICOM Structered Reporting. PixelMed Publishing, Pennsylvania (2000)

8. Silva, A.C.: Algoritmos para Diagnostico Assistido de Nodulos Pulmonares Solitarios em Imagens de Tomografia Computadorizada. PhD thesis, PUC-Rio (2004)

9. Gurcan, M.N., Sahiner, B., Petrick, N., Chan, H.P., Kazerooni, E.A., Cascade, P.N., Hadjiiski, L.M.: Lung nodule detection on thoracic computed tomography images: Preliminary evaluation of a computer-aided diagnosis system. Medical Physics 2552-2558 (2002)

10. Silva, A., Carvalho, P.C., Nunes, R., Gattass, M.: Algorithms for assisted diagnosis of solitary lung nodules in computerized tomography images. Technical Report TR2004-02, IMPA - Visgraf Laboratory (2004)

11. Burges, C.J.C.: A Tutorial on Support Vector Machines for Pattern Recognition. Kluwer Academic Publishers, Dordrecht (1998)

12. Chang, C.C., Lin, C.J.: LIBSVM: a library for support vector machines (2001), Available at http://www.csie.ntu.edu.tw/ cjlin/libsvm 